\section{Total body irradiation dose escalation decreases risk of progression and graft rejection after hematopoietic cell transplantation for myelodysplastic syndromes or myeloproliferative neoplasms}

Federico Monaco, ${ }^{1}$ Bart L. Scott, ${ }^{1,2}$ Thomas R. Chauncey, ${ }^{1,2,3}$ Finn B. Petersen ${ }^{4}$ Barry E. Storer, ${ }^{1,2}$ Frederic Baron, ${ }^{5}$ Mary E. Flowers, ${ }^{1,2}$ H. Joachim Deeg, ${ }^{1,2}$ David G. Maloney, ${ }^{1,2}$ Rainer Storb ${ }^{1,2}$ and Brenda M. Sandmaier ${ }^{1,2}$

${ }^{1}$ Fred Hutchinson Cancer Research Center, Seattle, WA, USA; 'University of Washington, Seattle, WA, USA; ${ }^{3}$ VA Puget Sound Health Care System, Seattle, WA, USA; " ${ }^{2}$ DS Hospital, Salt Lake City, UT, USA and ${ }^{5}$ University of Liege, Belgium

\section{ABSTRACT}

A non-myeloablative regimen of fludarabine and 200 cGy total body irradiation combined with post-grafting immunosuppression with mycophenolate mofetil and a calcineurin inhibitor facilitates allogeneic hematopoietic cell transplantation from HLA-matched related or unrelated donors in older patients and/or those with comorbidities. However, outcomes of prior studies have been disappointing in patients with myelodysplastic syndromes or myeloproliferative neoplasms due to high incidences of progression or graft failure (together termed hematopoietic cell transplantation-failure). We hypothesized that escalating the total body irradiation dose may improve the outcomes and subsequently performed a phase II total body irradiation dose-escalation trial. Patients with median age 66 years were enrolled in two arms to receive non-myeloablative conditioning followed by hematopoietic cell transplantation with total body irradiation dose escalation for excessive hematopoietic cell transplantation-failure: Arm A: myeloproliferative neoplasm/myelodysplastic syndrome low risk ( $\mathrm{n}=36$ ); and Arm B: myelodysplastic syndrome highrisk/chronic myelomonocytic leukemia $(n=41)$. Total body irradiation dose levels were: Level-1 (300 cGy), Level-2 (400 cGy), or Level-3 (450 cGy). Patients received intravenous fludarabine $30 \mathrm{mg} / \mathrm{m}^{2}$ for three days. Total body irradiation was administered on day 0 followed by infusion of peripheral blood stem cells from HLA-matched related $(n=30)$ or unrelated $(n=47)$ donors. Post-grafting immunosuppression with mycophenolate mofetil and cyclosporine was administered. The primary end point was day 200 hematopoietic cell transplant failure, with the objective of reducing the incidence to $<20 \%$. The primary end point was reached on Arm A at dose Level-1 (300 cGy total body irradiation) with a cumulative incidence of day 200 hematopoietic cell transplant failure of $11 \%$, and on Arm B at dose Level-3 (450 cGy) with a cumulative incidence of day 200 hematopoietic cell transplant failure of $9 \%$. Increasing the total body irradiation dose leads to a higher success rate with non-myeloablative conditioning by reducing relapse and rejection. Further studies are necessary to decrease non-relapse mortality, especially among patients with high-risk disease. Trial registered under clinicaltrials.gov identifier: NCT00397813.

\section{Introduction}

Hematopoietic cell transplantation (HCT) is the only curative option for patients with myelodysplastic syndromes (MDS) or myeloproliferative neoplasms (MPN). ${ }^{1}$ While these disorders are usually seen in older individuals, conventional HCT after
Ferrata Storti Foundation

Haematologica 2019

Volume 104(6):1221-1229

\section{Correspondence:}

BRENDA M. SANDMAIER

bsandmai@fredhutch.org

Received: June 7, 2018.

Accepted: January 2, 2019.

Pre-published: January 10, 2019.

doi:10.3324/haematol.2018.199398

Check the online version for the most updated information on this article, online supplements, and information on authorship \& disclosures: www.haematologica.org/content/104/6/1221

(C)2019 Ferrata Storti Foundation

Material published in Haematologica is covered by copyright. All rights are reserved to the Ferrata Storti Foundation. Use of published material is allowed under the following terms and conditions:

https://creativecommons.org/licenses/by-nc/4.0/legalcode. Copies of published material are allowed for personal or internal use. Sharing published material for non-commercial purposes is subject to the following conditions:

https://creativecommons. org//icenses/by-nc/4.0/legalcode sect. 3. Reproducing and sharing published material for commercial purposes is not allowed without permission in writing from the publisher. 
intensive cytotoxic conditioning regimen has been restricted to relatively young patients, without comorbidities. In order to address these limitations, we designed a non-myeloablative (NMA) regimen specifically for older patients and in those with comorbidities. This regimen consists of fludarabine (FLU) and 200 cGy total body irradiation (TBI) combined with post-grafting immunosuppression with mycophenolate mofetil (MMF) and a calcineurin inhibitor that facilitates allogeneic HCT with HLA-matched related (MRD) or unrelated donors (URD). Results of prior studies with this regimen have been very encouraging in most hematologic malignancies; ${ }^{2-5}$ however, in patients with chronic myelomonocytic leukemia (CMML) or previously untreated MDS/MPN, results have not been as successful. In a previous study, the cumulative incidence of HCT failure, defined as graft rejection or disease progression before day 200 after HCT, was $46 \%$ for the entire study population, $58 \%$ for patients with CMML or MDS with excess blasts (MDS-EB), and 31\% for patients with MDS [comprising MDS with single or multilineage dysplasia (MDS-SLD or MDS-MLD), MDS with ring sideroblasts (MDS-RS), MDS, unclassifiable (MDS-U) MDS with $<5 \%$ blasts], or MPN. The 200 -day, 1 -year and 2-year probabilities of progression-free survival (PFS) were $27 \%$, $19 \%$, and $11 \%$, respectively, in patients with CMML or MDS-EB, and $46 \%, 40 \%$, and $37 \%$, respectively, in patients with MDS with $<5 \%$ blasts or MPN.

Furthermore, it was shown that early achievement of full donor T-cell chimerism was associated with a reduced risk of relapse ( $\mathrm{HR} 0.5, P=0.002)$ in patients with hematologic malignancies receiving NMA conditioning. ${ }^{6}$

Taken together, these data suggest that increasing the intensity of the non-myeloablative conditioning regimen might delay disease progression until establishment of full donor chimerism and development of graft-versus-tumor (GvT) effects, thereby improving outcomes of patients with CMML, untreated MDS, and MPN.

\section{Methods}

\section{Study design}

A phase II TBI dose escalation trial (Figure 1) was conducted at Fred Hutchinson Cancer Research Center, Veterans Affairs Puget-Sound Health Care System, and Intermountain LDS Hospital. The Institutional Review Boards of the participating centers approved the protocol; all patients signed informed consent. An independent Data and Safety Monitoring Board oversaw the trial. The trial was registered at ClinicalTrials.gov, a service of the United States National Institutes of Health under number NCT00397813.

\section{Patients}

Participants were eligible if they had a World Health Organization defined diagnosis of CMML, MDS, MPN or paroxysmal nocturnal hemoglobinuria (PNH) with history of life-threatening complications.

Patients were $\geq 50$ and $<75$ years of age or were $<50$ years of age but at high risk for therapy-related toxicity from standard high-dose regimens; factors considered high risk included pre-existing conditions such as a chronic disease affecting kidneys, liver, lungs or heart, or previous failed HCT. Included patients were affected by MDS or MPN with less than $10 \%$ of blasts and not treated by myelosuppressive chemotherapy (defined as chemotherapy given with the intent of inducing a complete remission; hypomethylating agents and oral cytoreductive therapy were permitted). Patients with CMML who had progressed and received myelosuppressive chemotherapy could be enrolled if they had less than $5 \%$ of marrow blasts.

Donors were either HLA-MRD or URD; unrelated donors with only a single allele disparity for HLA-A, B, or $\mathrm{C}$ as defined by high-resolution typing were allowed. Only granulocyte-colony stimulating factor (G-CSF) mobilized peripheral blood stem cells (PBSC) were permitted as a HCT source.

\section{Conditioning regimens and immune suppression}

The conditioning regimens (Figure 2) were FLU (30 $\mathrm{mg} / \mathrm{m}^{2}$ /day IV) on days $-4,-3,-2$ and TBI of three magnitudes: 300 cGy (Level 1), 400 cGy (Level 2), or 450 cGy (Level 3) on day 0 at 6-7 cGy/min from linear accelerator, followed by HCT. For related recipients, graft-versus-host disease (GvHD) prophylaxis included cyclosporine (CSP) 5 $\mathrm{mg} / \mathrm{kg}$ PO every 12 hours from day -3 to day +56 with subsequent taper to day +180 and mycophenolate mofetil (MMF) at $15 \mathrm{mg} / \mathrm{kg}$ PO every 12 hours from day 0 to day +27 and then discontinued. For unrelated recipients, GvHD prophylaxis included CSP $5 \mathrm{mg} / \mathrm{kg}$ PO every 12 hours from day -3 to day +100 with taper to day +180 and $\mathrm{MMF}$ at $15 \mathrm{mg} / \mathrm{kg} \mathrm{PO}$ every eight hours from day 0 to day +40 and then tapering to day +96 . Growth factors were not given during the first 21 days after HCT; infection prophylaxis and transfusion support were as per institutional guidelines. Bone marrow aspirates or biopsies and chimerism analyses were performed on days $+28,+56$, +84 and +180 after HCT.

\section{Outcomes}

The purpose of this study was to evaluate whether a more intense but still NMA conditioning regimen could reduce the combined rates of graft rejection and disease progression (together termed HCT-failure) in this group of MDS and CMML patients, while maintaining an acceptable rate of non-relapse mortality (NRM).

The primary objective was to decrease the incidence of day-200 HCT-failure to $<20 \%$. HCT-failure in this study was defined as either progression of the underlying malignancy or graft failure. Progression was defined as recurrence of disease as detected by flow cytometry, morphology, cytogenetics, or mutation analysis. For patients with myelofibrosis, persistent fibrosis was not considered evidence of progression. Graft failure was defined as $<5 \%$ donor CD3 chimerism in the peripheral blood. Secondary objectives included PFS, NRM, and the kinetics of donor engraftment.

Primary cause of death was adjudicated using previously described criteria. ${ }^{7}$ When relapse occurred, it was considered the primary cause of death regardless of other events. Additionally, acute and chronic GvHD incidences were also evaluated. Grading of acute and chronic GvHD was performed according to the established recognized standards. ${ }^{8}$ The first day of acute GvHD of a certain grade was used to calculate cumulative incidence curves for that GvHD grade.

\section{Statistical analysis}

For purposes of this study, HCT-failure was defined as 
graft rejection (defined as $<5 \%$ donor $\mathrm{T}$-cell chimerism) or disease progression within 200 days of transplant.

Dose escalation was carried out independently in two groups of patients:

- Arm A: patients with MPN, low-risk MDS (MDS-SLD, MDS-MLD, MDS-U, MDS-RS) or PNH;

- Arm B: patients with high-risk MDS (MDS-EB-1) or CMML.

In each arm, up to 24 patients were accrued to each TBI dose level, in groups of 6 patients, with an escalation rule triggered for excessive HCT failure. If 24 patients were successfully enrolled at a TBI dose level without triggering the escalation rule for HCT failure (or other stopping rules), then that dose level would be considered a success and accrual would be closed for that arm. ARM A was modified after completing accrual of 24 patients at a dose level, to allow accrual of up to 12 additional patients at the same dose level while accrual to Arm B continued. Stopping rules for non-relapse mortality and TBI dose escalation continued to be monitored. Cohorts of 6 patients were only defined for purposes of determining dose escalation and stopping rules; the statistical analysis was based on the operating characteristics of a sequence of cohorts of 6 patients, not on single cohorts.

All patients received FLU $30 \mathrm{mg} / \mathrm{m}^{2} /$ day IV for three days preceding TBI.

The TBI dose levels were:

1. Dose Level 1: 300 cGy TBI

2. Dose Level 2: 400 cGy TBI

3. Dose Level 3: 450 cGy TBI

Dose escalation rules were imposed for HCT failure $>20 \%$ at day +200 . Stopping rules were imposed for NRM at day 200 of $>25 \%$ in Arm A and $>35 \%$ in Arm B. Overall survival (OS) and PFS were estimated by the Kaplan-Meier method. Cumulative incidences of relapse, NRM, and acute and chronic GvHD were estimated as previously described. ${ }^{9}$ All analyses were performed using SAS (SAS Institute, Cary, NC, USA).

\section{Results}

\section{Enrollment}

The study was opened on March 2006 and was closed to accrual on March 2017. Overall, 79 patients were screened, and 77 patients were enrolled. Patients' and

\section{NCT00397813 Flow Diagram}

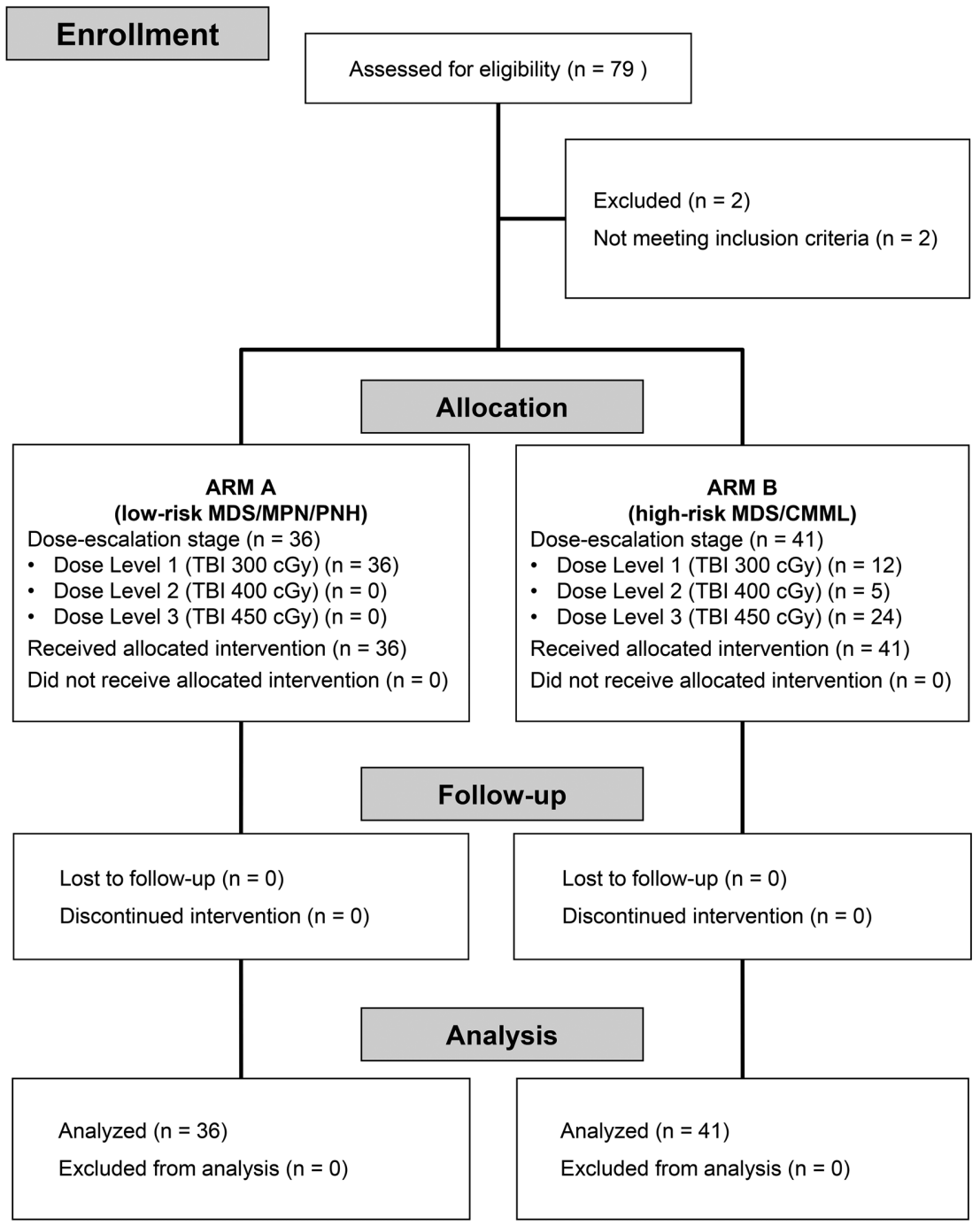

Figure 1. Flow diagram of the progress through the phases of the study. MDS: myelodysplastic syndrome; MPN: myeloproliferative neoplasm; PNH: paroxysmal nocturnal hemoglobinuria; CMML: chronic myelomonocytic leukemia; TBI: total body irradiation; cGy: centigray; n: number. 
transplant characteristics are summarized in Table 1. Thirty-six patients were enrolled in Arm A and 41 in Arm B.

\section{Engraftment}

Neutrophils declined below $0.5 \times 10^{9} / \mathrm{L}$ in 35 out of 36 patients in Arm A and in all patients in Arm B. The cumulative incidences of neutrophil recovery at day 28 were $100 \%$ in Arm A and $97.4 \%$ in Arm B. The median time to neutrophil $>0.5 \times 10^{9} / \mathrm{L}$ was 13.5 days in Arm $\mathrm{A}$ and 13 days in Arm B. The platelet counts did not decline below $20 \times 10^{9} / \mathrm{L}$ in 9 out of $36(25 \%)$ patients in Arm A and in 11 out of 41 (26.8\%) patients in Arm B. The cumulative incidences of platelet recovery $\left(>20 \times 10^{9} / \mathrm{L}\right)$ at day 84 were 97.2\% in Arm A and 100\% in Arm B. The median times to platelet recovery were 3.5 days in Arm A and 7 days in Arm B. There were two graft failures, both in patients enrolled in Arm A.

\section{Table 1. Patients' characteristics.}

\begin{tabular}{|c|c|c|}
\hline & ARM A $(n=36)$ & ARM B $(n=41)$ \\
\hline $\begin{array}{l}\text { Sex, n (\%) } \\
\text { Female } \\
\text { Male }\end{array}$ & $\begin{array}{c}9(25 \%) \\
27(75 \%)\end{array}$ & $\begin{array}{l}14(34.1 \%) \\
27(65.9 \%)\end{array}$ \\
\hline $\begin{array}{l}\text { Diagnosis, } \mathrm{n}(\%) \\
\text { Myelofibrosis } \\
\text { Low-risk MDS (MDS-SLD, MDS-MLD, MDS-U, MDS-RS) } \\
\text { PNH } \\
\text { High-risk MDS (MDS-EB-1) } \\
\text { CMML }\end{array}$ & $\begin{array}{c}18(50 \%) \\
17(47.2 \%) \\
1(2.8 \%)\end{array}$ & $\begin{array}{l}28(68.3 \%) \\
13(31.7 \%)\end{array}$ \\
\hline $\begin{array}{l}\text { Treatment, n (\%) } \\
\text { Dose level } 1 \text { (TBI } 300 \text { cGy) } \\
\text { Dose level } 2 \text { (TBI } 400 \text { cGy) } \\
\text { Dose level } 3 \text { (TBI } 450 \text { cGy) }\end{array}$ & $39(100 \%)$ & $\begin{array}{l}12(39.3 \%) \\
5(12.2 \%) \\
24(58.5 \%)\end{array}$ \\
\hline Time from diagnosis to HCT, month, median (range) & $15.4(4-336)$ & $8.4(1-92)$ \\
\hline Age at transplant, years, median (range) & $65(37-71)$ & $67(52-74)$ \\
\hline $\begin{array}{l}\text { HCT-CI, n (\%) } \\
0 \\
1-2 \\
\geq 3\end{array}$ & $\begin{array}{c}\text { Median } 3 \text { (range 0-8) } \\
4(11.1 \%) \\
11(30.6 \%) \\
21(58.3 \%)\end{array}$ & $\begin{array}{c}\text { Median } 3 \text { (range 0-9) } \\
7(17.1 \%) \\
12(29.3 \%) \\
22(53.6 \%)\end{array}$ \\
\hline
\end{tabular}

\section{Previous stem-cell transplantation, $\mathrm{n}$}

Autologous

Allogeneic

Donor, n (\%)

0

MRD
URD $10 / 10$

URD $9 / 10$

Neutrophils $<0.5 \times 10^{9} / \mathrm{L}$, days, median (range)

Platelets $<20 \times 10^{9} \mathrm{~L}$, days, median (range)

HCT-failure before day $+200, n$

Relapse

Graft failure

Relapse, $\mathrm{n}$

Death, $n$

Cause of death, $n$

Progression/relapse

NRM non-GvHD related
NRM GvHD related

(n)

(2)

$16(44.4 \%)$
$18(50 \%)$
$2(5.6 \%)$
$13.5(0-28)$
$3.5(0-107)$

\section{$14(34.1 \%)$ \\ $25(61 \%)$ \\ $2(4.9 \%)$}

$13(2-29)$

$7(0-43)$

$\begin{array}{cc}3 & 10 \\ 2 & \\ 9 & 15 \\ 24 & 31\end{array}$

10

31

\section{5}

6

Pneumonia $\mathrm{n}=2$

Pneumonia $\mathrm{n}=3$

Sepsis $\mathrm{n}=1$

Disseminated aspergillus $n=1$

Suicide $n=1$

9

GvHD refractory $\mathrm{n}=4$

Pneumonia $\mathrm{n}=2$

Multiorgan failure $\mathrm{n}=1$

CNS bleeding $n=1$

Sepsis $n=1$

Follow up, months, median (range)

69.1 (39.1-121.8)

Cardiac toxicity $n=2$

Multiorgan failure $\mathrm{n}=2$

\section{0}

GvHD refractory $n=3$

Pneumonia $n=2$

Sepsis $n=4$

Disseminated aspergillus $n=1$

21 (3.4-102.6)

CMML: chronic myelomonocytic leukemia; CNS: central nervous system; GvHD: graft-versus-host disease; HCT-CI: hematopoietic cell transplantation comorbidity index; MDS: myelodysplastic syndrome; MDS-EB myelodysplastic syndrome with excess of blasts-1; MDS-RS: myelodysplastic syndrome with ring sideroblast; MDS-U: myelodysplastic syndrome unclassifiable; MLD: multilineage dysplasia; MRD: HLA-matched related donor; NRM: non-relapse mortality; PNH: paroxysmal nocturnal hemoglobinuria; SLD: single lineage dysplasia; TBI: total body irradiation; URD: HLA-matched unrelated donor. 


\section{Chimerism analysis}

In Arm A, the day 28 median donor chimerism levels were $68 \%$ for $\mathrm{CD} 3$ and $100 \%$ for $\mathrm{CD} 33$, and the day 84 levels were $79 \%$ and $100 \%$, respectively. In Arm B, for patients who received TBI at dose Levels-1 or 2 (300 cGy or $400 \mathrm{cGy}$ ), the day 28 median donor chimerism levels were $84 \%$ for $\mathrm{CD3}$ and $77 \%$ for $\mathrm{CD} 33$, and the day 84 levels were $85 \%$ and $100 \%$, respectively. For patients who received TBI at dose Level-3 (450 cGy) in Arm B, the day 28 median donor chimerism levels were $82 \%$ for $\mathrm{CD} 3$ and $100 \%$ for $\mathrm{CD} 33$, and the day 84 levels were $81 \%$ and $100 \%$, respectively.

Hematopoietic cell transplantation failure and relapse

In Arm A, 4 out of 36 patients given TBI at 300 cGy had HCT-failure before day 200, three due to progression and one to graft rejection, with a cumulative incidence of $11 \%$. The escalation rule in this arm was not reached so all patients received dose Level-1 with TBI at 300 cGy. In Arm B, 12 patients received dose Level-1 with TBI at 300 cGy and 5 had HCT-failure, all due to progression, triggering the TBI dose escalation rule. The next five patients received dose Level-2 TBI (400 cGy) and three experienced HCT-failure, all due to progression, triggering again the dose escalation rule. The cumulative incidence of HCT-failure for dose Level-1 and dose Level-2 combined was $47 \%$. The subsequent 24 patients received dose Level-3 with TBI at 450 cGy and only two experienced HCT-failure before 200 days, with a cumulative incidence of $9 \%$. The patients' characteristics and outcomes for the high-risk patients enrolled on Arm B (separated by TBI dose) are shown in Online Supplementary Table S1 (Table 2 and Figure 3A and E).
The cumulative incidences of progression in Arm A at one year, two years, and five years was $17 \%, 19 \%$, and $22 \%$, respectively. Patients who received TBI at dose Levels-1 or 2 (300 cGy or 400 cGy) had cumulative incidences of progression at one year, two years, and five years of $53 \%, 53 \%$ and $53 \%$, respectively. Patients who received TBI at dose Level-3 (450 cGy) in Arm B had cumulative incidences of progression at one year, two years, and five years of $24 \%, 24 \%$ and $32 \%$, respectively.

\section{Graft-versus-host disease}

The cumulative incidences of grades II-IV and grade III-IV acute GvHD at day 100 were $58 \%$ and $13 \%$, respectively. The cumulative incidences of chronic GvHD at one year and two years were $38 \%$ and $45 \%$, respectively: one, 21, and eight patients developed mild, moderate, and severe chronic GvHD, respectively. No statistically significant differences were seen between each arm with regards to incidence of acute or chronic GvHD (cumulative incidences in each arm are shown in Table 2) (Figure 4).

\section{Non-relapse mortality}

The cumulative incidences of NRM in Arm A at one year, two years, and five years were $17 \%, 22 \%$, and $43 \%$, respectively. Patients who received TBI at dose Levels-1 or 2 (300 cGy or 400 cGy) had cumulative incidences of NRM at one year, two years, and five years of $29 \%, 35 \%$, and $35 \%$, respectively. Patients in Arm B who received TBI at dose Level-3 (450 cGy) had cumulative incidences of NRM at one year, two years, and five years of $31 \%$, $43 \%$, and $51 \%$, respectively (Table 2 and Figure $3 \mathrm{D}$ ).

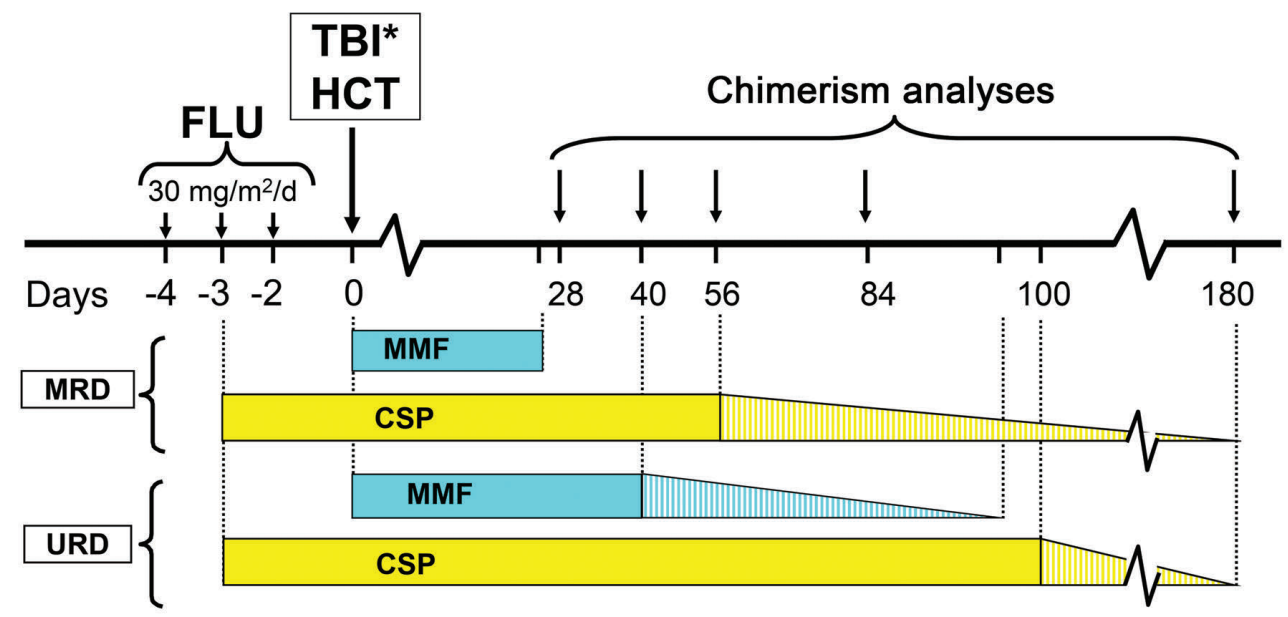

FLU: $30 \mathrm{mg} / \mathrm{m}^{2} \times 3$ days (days $-4,-3,-2$ ).

TBI: Single fraction at $7 \mathrm{cGy} / \mathrm{min}$. $300 \mathrm{cGy}$ (level 1), $400 \mathrm{cGy}$ (level 2), $450 \mathrm{cGy}$ (level 3).

CSP: MRD: $5.0 \mathrm{mg} / \mathrm{kg}$ po $\mathrm{q} 12 \mathrm{hrs}$ days -3 to +56 , then taper to day +180 .

URD: $5.0 \mathrm{mg} / \mathrm{kg}$ po q12 hrs days -3 to +100 , then taper to day +180 .

MMF: MRD: $15 \mathrm{mg} / \mathrm{kg}$ po $\mathrm{q} 12$ hrs days 0 to +27 .

URD: $15 \mathrm{mg} / \mathrm{kg}$ po $\mathrm{q} 8 \mathrm{hrs}$ days 0 to +40 , then taper to day +96 .

Figure 2. Outline of treatment plan involving conditioning regimens and graft-versus-host disease (GvHD) prophylaxis. FLU: fludarabine; TBI: total body irradiation; HCT: hematopoietic cell transplantation; MRD: HLA-matched related donor; URD: HLA-matched unrelated donor; MMF: mycophenolate mofetil; CSP: cyclosporine; cGy: centigray; hrs: hours. 


\section{Causes of death}

Twenty-four of 36 patients in Arm A died; GvHD and disease progression were the primary causes of death for nine patients each. In Arm B, 31 out of 41 patients died; progression was the primary cause of death for 15 patients (Table 1).

\section{Overall survival and progression-free survival}

In Arm A, OS at one year, two years, and five years were $72 \%, 61 \%$, and $38 \%$, respectively, and PFS were $68 \%, 58 \%$, and $35 \%$, respectively. Patients who received TBI at dose Levels 1 or 2 (300 cGy or 400 cGy) in Arm B had OS at one year, two years, and five years of $24 \%$, $12 \%$, and $12 \%$, respectively, and PFS of $18 \%, 12 \%$, and $12 \%$, respectively. Patients in Arm B who received TBI at dose Level-3 (450 cGy) had OS at one year, two years, and five years of $60 \%, 37 \%$, and $22 \%$, respectively, and PFS of $45 \%, 33 \%$, and $17 \%$, respectively (Table 2 and Figure $3 \mathrm{~B}$ and $\mathrm{C})$.

\section{Discussion}

In the early 2000s, at Fred Hutchinson Cancer Research Center, a regimen for NMA conditioning using the combination of fludarabine and 200 cGy TBI was developed. This regimen allowed for donor cell engraftment but was characterized by a high incidence of early disease progression or graft rejection, ${ }^{2.5}$ especially for high-risk patients. In 2006, Scott et al. ${ }^{10}$ published results on 38 patients affected by MDS or acute myeloid leukemia (AML) who received an NMA regimen with 3-year rates of OS, PFS, and NRM of $27 \%, 28 \%$, and $41 \%$, respectively. These results were retrospectively compared with patients who underwent myeloablative conditioning, and no statistically significant differences were seen. Disease progression was the main issue with a cumulative incidence of $31 \%$ at three years. Laport et al..$^{11}$ reported data from our retrospective study on 148 patients diagnosed with MDS or MPN who underwent allogeneic HCT after NMA conditioning. PFS and OS at three years were both $27 \%$ for all patients, with a progression incidence of $41 \%$. When stratifying data, PFS rates at three years for patients with de novo MDS ( $\mathrm{n}=40)$, treatment-related MDS $(n=25), M P N(n=27)$, and CMML $(n=7)$ were $22 \%, 29 \%, 37 \%$, and $43 \%$, respectively, and OS rates were $20 \%, 27 \%, 43 \%$ and $43 \%$, respectively. For all patients, NRM at three years was $32 \%$. In both analyses, disease progression was the major cause of treatment failure and the leading cause of death; it appears that this regimen may not have conferred enough cytoreduction for adequate disease control. The data from these retrospective reviews formed the basis for the current study.

Cliff et al. ${ }^{12}$ had previously shown that an escalation in TBI dose to 15.75 Gy from 12 Gy reduced relapse/progression $(13 \%$ vs. $35 \%)$. This result demonstrated that a decrease in tumor burden before transplantation led to a decreased risk of disease progression; however, most patients with MDS or MPN are not candidates for myeloablative TBI conditioning regimens either due to advanced age or comorbidities.

Taking into consideration these data, along with results from pre-clinical studies, ${ }^{13,14}$ we hypothesized that raising

Table 2. Results.

\begin{tabular}{|c|c|c|c|c|}
\hline & $\%$ at 100 days & $\%$ at 1 year & $\%$ at 2 years & $\%$ at 5 years \\
\hline $\begin{array}{l}\text { Overall survival } \\
\text { Arm A TBI dose Level-1(300 cGy) } \\
\text { Arm B TBI dose Level-1 or } 2 \text { (300-400 cGy) } \\
\text { Arm B TBI dose Level-3 (450 cGy) }\end{array}$ & & $\begin{array}{l}72 \\
24 \\
60\end{array}$ & $\begin{array}{l}61 \\
12 \\
37\end{array}$ & $\begin{array}{l}38 \\
12 \\
22\end{array}$ \\
\hline $\begin{array}{l}\text { Progression-free survival } \\
\text { Arm A TBI dose Level-1(300 cGy) } \\
\text { Arm B TBI dose Level-1 or } 2 \text { (300-400 cGy) } \\
\text { Arm B TBI dose Level-3 (450 cGy) }\end{array}$ & & $\begin{array}{l}68 \\
18 \\
45\end{array}$ & $\begin{array}{l}58 \\
12 \\
33\end{array}$ & $\begin{array}{l}35 \\
12 \\
17\end{array}$ \\
\hline $\begin{array}{l}\text { Relapse incidence } \\
\text { Arm A TBI dose Level-1(300 cGy) } \\
\text { Arm B TBI dose Level-1 or } 2 \text { (300-400 cGy) } \\
\text { Arm B TBI dose Level-3 (450cGy) }\end{array}$ & & $\begin{array}{l}17 \\
53 \\
24\end{array}$ & $\begin{array}{l}19 \\
53 \\
24\end{array}$ & $\begin{array}{l}22 \\
53 \\
32\end{array}$ \\
\hline $\begin{array}{l}\text { Non-relapse mortality } \\
\text { Arm A TBI dose Level-1(300 cGy) } \\
\text { Arm B TBI dose Level-1 or } 2 \text { (300-400 cGy) } \\
\text { Arm B TBI dose Level-3 (450 cGy) }\end{array}$ & & $\begin{array}{l}17 \\
29 \\
31\end{array}$ & $\begin{array}{l}22 \\
35 \\
43\end{array}$ & $\begin{array}{l}43 \\
35 \\
51\end{array}$ \\
\hline $\begin{array}{l}\text { Chronic GvHD } \\
\text { Arm A TBI dose Level-1(300 cGy) } \\
\text { Arm B TBI dose Level-1 or } 2 \text { (300-400 cGy) } \\
\text { Arm B TBI dose Level-3 (450 cGy) }\end{array}$ & & 38 & $\begin{array}{l}45 \\
53 \\
35 \\
40\end{array}$ & \\
\hline $\begin{array}{l}\text { Acute GvHD (grades 2-4) } \\
\text { Arm A TBI dose Level-1(300 cGy) } \\
\text { Arm B TBI dose Level-1 or } 2 \text { (300-400 cGy) } \\
\text { Arm B TBI dose Level-3 (450 cGy) }\end{array}$ & $\begin{array}{l}58 \\
56 \\
65 \\
57\end{array}$ & & & \\
\hline $\begin{array}{l}\text { Acute GvHD (grades 3-4) } \\
\text { Arm A TBI dose Level-1(300 cGy) } \\
\text { Arm B TBI dose Level-1 or } 2 \text { (300-400 cGy) } \\
\text { Arm B TBI dose Level-3 (450 cGy) }\end{array}$ & $\begin{array}{c}13 \\
17 \\
12 \\
9\end{array}$ & & & \\
\hline
\end{tabular}

TBI: total body irradiation; GvHD: graft-versus-host disease. 
the TBI dose from the originally used 200 cGy could improve the outcome of HCT in the NMA setting, thereby reducing the risk of disease progression in patients who are not considered candidates for myeloablative conditioning.

In agreement with this hypothesis, we showed improvement in outcome by raising the TBI dose from 200 cGy to 300 cGy in our cohort of low-risk patients (MDS-SLD, MDS-MLD, MDS-RS, MDS-U and MPN). Similarly, the day 200 failure rate for high-risk patients (MDS-EB-1/CMML) was reduced to $9 \%$ when TBI was raised to $450 \mathrm{cGy}$ while the lower TBI doses of $300 \mathrm{cGy}$ and 400 cGy failed to improve results. We achieved the primary objective of reducing transplant failure to less than $20 \%$ by day 200 , which resulted in PFS at two years of $58 \%$ in the low-risk group and $33 \%$ in the high-risk group. NRM was not significantly increased after escalating TBI to $300 \mathrm{cGy}$, but $450 \mathrm{cGy}$ TBI led to toxicity with a resultant NRM of $43 \%$ at two years after HCT. Limitations of the current study include the slow accrual over ten years. While donor selection and GvHD prophylaxis remained unchanged during this interval, and there were no major changes in antimicrobial treatments, radiological techniques and ancillary therapies, incremental

A

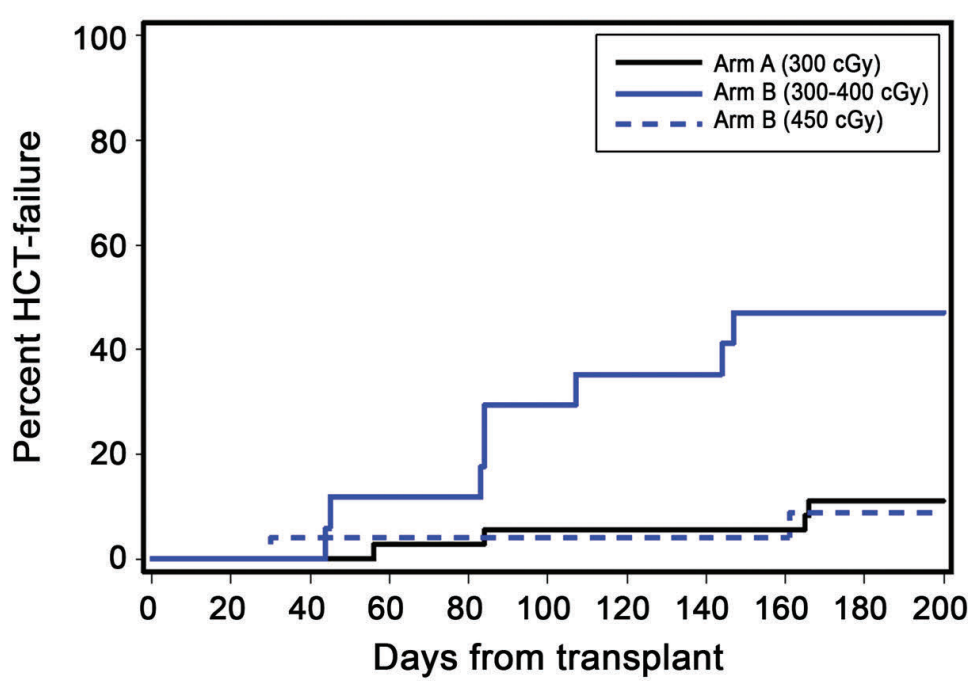

B

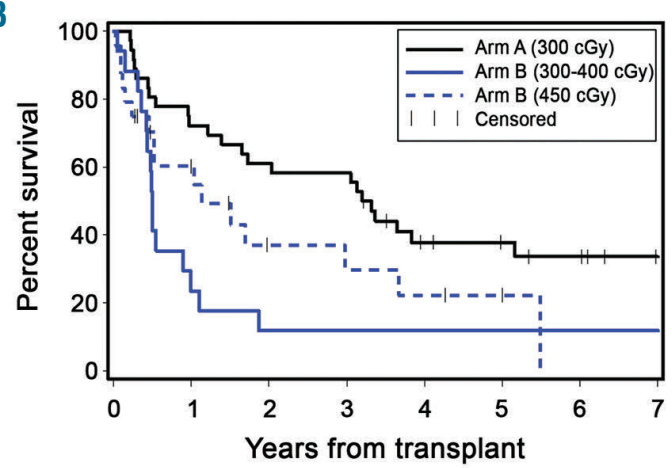

D

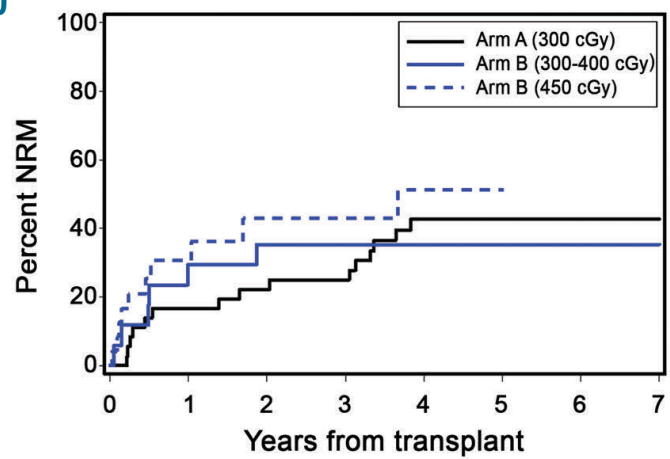

C

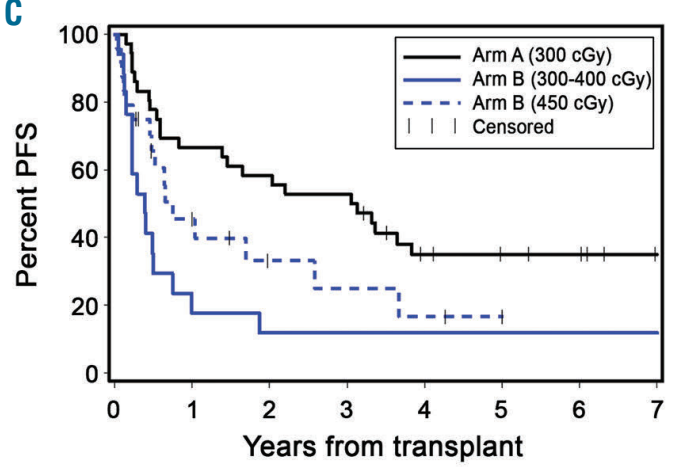

$E$

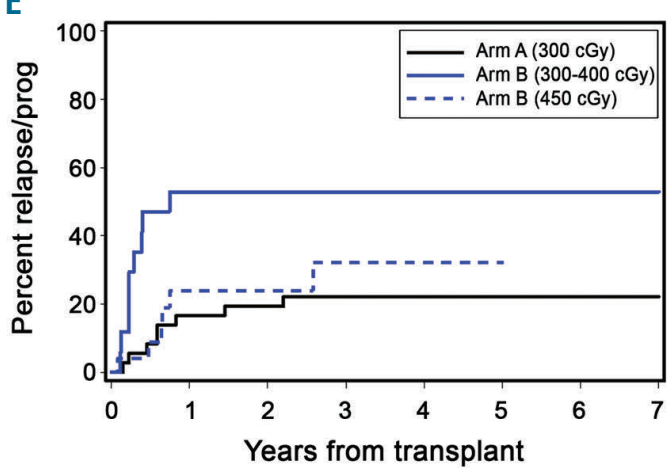

Figure 3. Patients enrolled in Arm A receiving 300 cGy total body irradiation (TBI) and patients enrolled in Arm B receiving $450 \mathrm{cGy}$ TBI had reduced day 200 hematopoietic cell transplantation (HCT) failure. Transplant outcomes by Arm and TBI dose: (A) HCT-failure, (B) overall survival, (C) progression-free survival (PFS), (D) non-relapse mortality (NRM), and (E) relapse incidence. 
improvements could be a bias. The slow accrual was due to competing protocols at our Centers. Another limitation regards high-risk patients who received dose level-2 TBI (400 cGy). Only five patients were enrolled but, as per protocol design, we met dose escalation rules because of transplant failure. It is possible that, with greater numbers, better results could be achieved.

The incidence of non-relapse mortality remains an issue, being high especially for high-risk patients (cumulative incidence of $43 \%$ at 2 years). There was no statistically significant difference regarding TBI-dose and GvHD incidence. Different strategies are needed to reduce the toxicity of 450 cGy TBI. Fractionation of the TBI dose was investigated in two preclinical canine experiments, which showed that fractionation of $450 \mathrm{cGy}$ TBI was significantly less immunosuppressive with a higher rate of graft rejection than when given as a single dose. ${ }^{15,16}$ Stelljes et al. demonstrated that a conditioning regimen of $8 \mathrm{~Gy}$ fractionated TBI and FLU was feasible with low NRM in patients with AML (8\% at 2 years for patients in CR, 37\% at 2 years for patients with active disease). ${ }^{17} \mathrm{~A}$ novel approach to replace or augment TBI while reducing toxicity, consists of using targeted radioimmunotherapy. ${ }^{18}$ Monoclonal antibodies to CD45 and CD20 coupled to beta $(\beta)$-emitting radionuclides (iodine-131 or yttrium-90) have been studied ${ }^{19,20}$ showing good results but evident disadvantages (e.g. off-target effects and the need to use isolation rooms) in clinical trials. However, the recent substitution with alpha $(\alpha)$-emitting radionuclides (bismuth213 and astatine-211) has given encouraging results, ${ }^{21,22}$ especially for astatine-211. ${ }^{23}$ Clinical trials are currently in progress using astatine-211 (clinicaltrials.gov identifier: NCT03128034).

The high incidence of GvHD irrespective of TBI dose remains a problem, especially for grades III-IV acute $\mathrm{GvHD}$ and chronic GvHD that reached cumulative incidences of $13 \%$ at 200 days and $45 \%$ at two years. These complications impacted OS and NRM, accounting for one-third of all deaths. Novel approaches are currently being investigated that are aimed at reducing GvHD incidence: the triple-drug combination using tacrolimus, mycophenolate mofetil, and sirolimus has been studied with encouraging results, ${ }^{24}$ and a phase III trial evaluated the addition of sirolimus to cyclosporine and $M M F$ in order to reduce GvHD without impairing graft-versus-tumor effect with significantly less acute GvHD and less NRM with superior overall and progression-free survival (clinicaltrials.gov identifier: NCT01251575). ${ }^{25}$

Lim et al. ${ }^{26}$ recently described results from a large retrospective study by the European Group for Blood and Marrow Transplantation (EBMT) registry which included 1,333 patients with MDS, who were older than 50 years, of whom 833 (62\%) underwent reduced intensity conditioning (RIC). The RIC regimens included FLU plus intermediate doses of one or two alkylating agents including busulfan, melphalan, cyclophosphamide, or thiotepa, or low-dose TBI (200-400 cGy) with or without anti-thymocyte globulin or alemtuzumab. OS, NRM and relapse incidence (RI) rates at four years were $32 \%, 32 \%$ and $41 \%$, respectively. The median age of patients undergoing RIC was 59 years (range 50-74.7), which was younger than current patients, and no comorbidity data was reported. Similarly, McClune et al. ${ }^{27}$ performed another large retrospective analysis of data of the Center for International
Blood and Marrow Transplant Research (CIBMTR) involving 1,080 patients of whom 535 had MDS and received an RIC or NMA conditioning regimen before transplantation. Of this cohort, 181 patients were over 60 years of age but, again, no comorbidity scores were reported. At two years after HCT, results for patients 60 64 years of age or over 65 years old included OS 45\% and $38 \%$, RFS $35 \%$ and $36 \%$, NRM $35 \%$ and $39 \%$, and relapse rate of $29 \%$ and $21 \%$, respectively. Recently Scott et $a .^{28}$ concluded a randomized prospective trial showing that more intensive conditioning might be better than RIC for patients with AML or MDS. A reduction in NRM for RIC (4.4\% vs. $15.8 \%$ at 18 months) was offset by increased disease progression $(37 \%$ vs. $3.7 \%$ at 18 months), although there were no statistically significant differences in OS and relapse-free survival between the two groups of patients.

Storb and Sandmaier ${ }^{29}$ analyzed results of eleven different retrospective studies using RIC or NMA regimens for various hematologic diseases reported by registries or individual transplant centers on nearly 9,000 patients. With a median follow up of three years (range 1.75-5), the incidence of disease progression was $43 \%$ (range 22 $65 \%$ ), NRM 34\% (range 6-38\%), and OS 38\% (22-65\%).

Non-myeloablative conditioning has extended the use of allogeneic HCT for patients with myeloid malignancies who were previously not considered candidates for HCT because of advanced age or comorbidities. Initial results with a regimen based on 200 cGy TBI was associated with a relatively

A

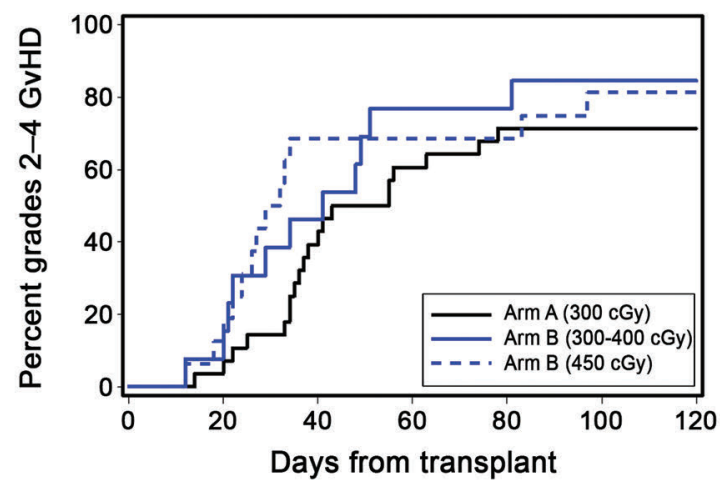

B

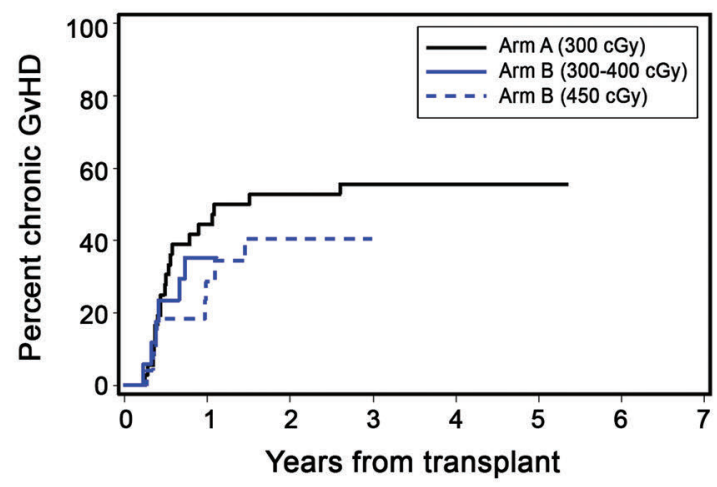

Figure 4. Incidences of graft-versus-host disease (GvHD) were comparable between different Arms and TBI doses. (A) Acute GvHD, (B) chronic GvHD. 
high rate of disease progression or relapse. We showed that an incremental increase in TBI resulted in a lower risk of disease progression/relapse among patients with MDS, MPN, and CMML. However, the escalation of the TBI dose to 450 cGy was associated with increased NRM, offsetting the benefit achieved from decreased disease recurrence. Future studies will, therefore, employ targeted radioimmunotherapy in order to reduce relapse/progression without added toxicity and without increasing NRM.

\section{Acknowledgments}

We thank the patients who have participated in this trial along with the clinical staff and research staff who assisted in the con- duct of the trial. We also thank Helen Crawford for help with manuscript preparation and submission.

\section{Funding}

Support for this study was provided from grants CA078902 from National Cancer Institute and grant HL36444 from the National Heart, Lung and Blood Institute, National Institutes of Health. This work was also in part supported by the NIH/NCI Cancer Center Support Grant P3O CA015704. The content is solely the responsibility of the authors and does not necessarily represent the official views of the National Cancer Institute or the National Institutes of Health nor their subsidiary Institutes and Center.

\section{References}

1. Scott B, Deeg HJ. Hemopoietic cell transplantation as curative therapy of myelodysplastic syndromes and myeloproliferative disorders. Best Pract Res Clin Haematol. 2006;19(3):519-522.

2. McSweeney PA, Niederwieser D, Shizuru JA, et al. Hematopoietic cell transplantation in older patients with hematologic malignancies: replacing high-dose cytotoxic therapy with graft-versus-tumor effects. Blood. 2001;97(11):3390-3400.

3. Niederwieser D, Maris M, Shizuru JA, et al. Low-dose total body irradiation (TBI) and fludarabine followed by hematopoietic cell transplantation (HCT) from HLA-matched or mismatched unrelated donors and postgrafting immunosuppression with cyclosporine and mycophenolate mofetil (MMF) can induce durable complete chimerism and sustained remissions in patients with hematological diseases. Blood. 2003;101(4):1620-1629.

4. Maris MB, Niederwieser D, Sandmaier BM, et al. HLA-matched unrelated donor hematopoietic cell transplantation after nonmyeloablative conditioning for patients with hematologic malignancies. Blood. 2003;102(6):2021-2030.

5. Sandmaier BM, Maris M, Maloney DG, et al. Low-dose total body irradiation (TBI) conditioning for hematopoietic cell transplants (HCT) from HLA-matched related (MRD) and unrelated (URD) donors for patients with hematologic malignancies: a five-year experience. Blood. 2003; 102(11):78-79.

6. Baron F, Maris MB, Sandmaier BM, et al. Graft-versus-tumor effects after allogeneic hematopoietic cell transplantation with nonmyeloablative conditioning. J Clin Oncol. 2005;23(9):1993-2003.

7. Copelan E, Casper JT, Carter SL, et al. A scheme for defining cause of death and its application in the T cell depletion trial. Biol Blood Marrow Transplant. 2007; 13 (12):1469-1476.

8. Glucksberg H, Storb R, Fefer A, et al. Clinical manifestations of graft-versus-host disease in human recipients of marrow from HL-Amatched sibling donors. Transplantation. 1974;18(4):295-304.

9. Gooley TA, Leisenring W, Crowley J, Storer BE. Estimation of failure probabilities in the presence of competing risks: new representations of old estimators. Stat Med. 1999;18(6):695-706.

10. Scott BL, Sandmaier BM, Storer B, et al Myeloablative vs nonmyeloablative allo- geneic transplantation for patients with myelodysplastic syndrome or acute myelogenous leukemia with multilineage dysplasia: a retrospective analysis. Leukemia. 2006;20(1):128-135.

11. Laport GG, Sandmaier BM, Storer BE, et al. Reduced-intensity conditioning followed by allogeneic hematopoietic cell transplantation for adult patients with myelodysplastic syndrome and myeloproliferative disorders. Biol Blood Marrow Transplant. 2008;14(2):246-255.

12. Clift RA, Buckner CD, Appelbaum FR, et al. Allogeneic marrow transplantation in patients with acute myeloid leukemia in first remission: A randomized trial of two irradiation regimens. Blood. 1990; 76(9):18671871.

13. Storb R, Yu C, Wagner JL, et al. Stable mixed hematopoietic chimerism in DLA-identical littermate dogs given sublethal total body irradiation before and pharmacological immunosuppression after marrow transplantation. Blood. 1997;89(8): 3048-3054

14. Hogan WJ, Little MT, Zellmer E, et al. Postgrafting immunosuppression with sirolimus and cyclosporine facilitates stable mixed hematopoietic chimerism in dogs given sublethal total body irradiation before marrow transplantation from DLA-identical littermates. Biol Blood Marrow Transplant. 2003;9(8):489-495.

15. Storb R, Raff RF, Appelbaum FR, et al. Comparison of fractionated to single-dose total body irradiation in conditioning canine littermates for DLA-identical marrow grafts. Blood. 1989;74(3):1139-1143.

16. Storb R, Raff RF, Appelbaum FR, et al. Fractionated versus single-dose total body irradiation at low and high dose rates to condition canine littermates for DLA-identical marrow grafts. Blood. 1994;83(11): 33843389.

17. Stelljes M, Bornhauser M, Kroger M, et al. Conditioning with 8-Gy total body irradiation and fludarabine for allogeneic hematopoietic stem cell transplantation in acute myeloid leukemia. Blood. 2005; 106(9):3314-3321.

18. Appelbaum FR, Brown P, Sandmaier B, et al. Antibody-radionuclide conjugates as part of a myeloblative preparative regimen for marrow transplantation. Blood. 1989; 73(8):2202-2208.

19. Pagel JM, Gooley TA, Rajendran J, et al. Allogeneic hematopoietic cell transplantation after conditioning with 131 I-antiCD45 antibody plus fludarabine and lowdose total body irradiation for elderly patients with advanced acute myeloid leukemia or high-risk myelodysplastic syn- drome. Blood. 2009;114(27):5444-5453

20. Gopal AK, Guthrie KA, Rajendran J, et al. 90 Y-Ibritumomab tiuxetan, fludarabine, and TBI-based nonmyeloablative allogeneic transplantation conditioning for patients with persistent high-risk B-cell lymphoma. Blood. 2011;118(4):1132-1139.

21. Sandmaier BM, Bethge WA, Wilbur DS, et al. Bismuth 213-labeled anti-CD45 radioimmunoconjugate to condition dogs for nonmyeloablative allogeneic marrow grafts. Blood. 2002;100(1):318-326.

22. Chen Y, Kornblit B, Hamlin DK, et al. Durable donor engraftment after radioimmunotherapy using alpha-emitter astatine211-labeled anti-CD45 antibody for conditioning in allogeneic hematopoietic cell transplantation. Blood. 2012;119(5):11301138.

23. Nakamae H, Wilbur DS, Hamlin DK, et al. Biodistribution, myelosuppression, and toxicities in mice treated with an anti-CD45 antibody labeled with the $\alpha$-emitting radionuclides bismuth-213 or astatine- 211 . Cancer Res. 2009;69(6):2408-2415.

24. Kornblit B, Maloney DG, Storer BE, et al. A randomized phase II trial of tacrolimus, mycophenolate mofetil and sirolimus after non-myeloablative unrelated donor transplantation. Haematologica. 2014; 99(10): 1624-1631.

25. Sandmaier BM, Maloney DG, Storer BE, et al. Sirolimus combined with mycophenolate mofetil (MMF) and cyclosporine (CSP) significantly improves prevention of acute graft-versus-host-disease (GVHD) after unrelated hematopoietic cell transplantation (HCT): Results from a phase III randomized multi-center trial. Blood. 2016;128(22):506.

26. Lim Z, Brand R, Martino R, et al. Allogeneic hematopoietic stem-cell transplantation for patients 50 years or older with myelodysplastic syndromes or secondary acute myeloid leukemia. J Clin Oncol. 2010; 28(3):405-411.

27. McClune BL, Weisdorf DJ, Pedersen TL, et al. Effect of age on outcome of reducedintensity hematopoietic cell transplantation for older patients with acute myeloid leukemia in first complete remission or with myelodysplastic syndrome. J Clin Oncol. 2010;28(11):1878-1887.

28. Scott BL, Pasquini MC, Logan BR, et al. Myeloablative versus reduced-intensity hematopoietic cell transplantation for acute myeloid leukemia and myelodysplastic syndromes. J Clin Oncol. 2017;35(11):11541161

29. Storb R, Sandmaier BM. Nonmyeloablative allogeneic hematopoietic cell transplantation. Haematologica. 2016;101(5):521-530. 